\title{
Características clínicas y metabólicas de los estados de intolerancia a la glucosa y glicemia de ayuno alteradas
}

\author{
Antonio Arteaga, Felipe Pollak, Leonor Robres, \\ Nicolás Velasco.
}

\section{Clinical and metabolic features of subjects with glucose intolerance and high fasting glucose levels}

Background: Subjects with glucose intolerance or high fasting glucose levels have a higher cardiovascular risk and frequently become diabetic. Aim: To assess clinical and metabolic characteristics of patients with glucose intolerance or high fasting glucose levels. Material and methods: Fasting and post glucose load serum glucose and insulin levels were measured in 1404 people, aged 42,0 $\pm 14,2$ years ( $81 \%$ women) with high diabetic risk. We categorized subjects in different alterations of blood glucose, according to 2006 American Diabetes Association categories. Insulin resistance (RI), insulin secretion ( $\Omega$ $\%$ ) and insulin disposition (ID), were calculated using fasting blood glucose and insulin levels, using the homeostasis model assessment (HOMA I and II). Results: Sixty percent of studied subjects had first grade relatives with diabetes mellitus and 1097 (78\%) were categorized as normal $(\mathrm{N}), 45(3 \%)$ as Diabetes Mellitus (DM), $161(11 \%)$ as high fasting glucose levels (GAA) and 103 (7\%) as glucose intolerant (ITG). Fifty three of the 106 subjects with GAA (50\%), were also glucose intolerant. Subjects with GAA had similar insulin sensitivity and lower $\beta$ cell function than $\mathrm{N}$ (insulin disposition $58 \pm 12$ and $111 \pm 32 \%$, respectively, $\mathrm{p}<0.01$ ). ITG had less insulin sensitivity than N (HOMA-IR $2.6 \pm 1.50 \pm$ and 2.0 \pm 1.30 , respectively) and only a mild decrease in $\beta$ cell function (insulin disposition $96 \pm 26$ and $111 \pm 32 \%$ respectively, $p<0.01$ ). Patients GAA plus ITG had similar alterations than those with DM (HOMA-IR $3.8 \pm 2.2$ and $4.4 \pm 3.7$ respectively; insulin disposition $57 \pm 10$ and $56.0 \pm 26 \%$ respectively. Conclusions: Patients with higher fasting glucose levels behave differently from those with glucose intolerance. High fasting glucose levels are highly prevalent in subjects with high risk of DM and must be considered as risk indicator in preventive programs for diabetes mellitus (Rev Méd Chile 2009; 137: 193-9).

(Key words: Glucose intolerance; Insulin; Insulin resistance) 
$\mathrm{D}$ eterminadas condiciones ambientales más una predisposición genética incrementan la resistencia a la insulina, lo que, asociado a un progresivo deterioro de la función de las células $\beta$ del páncreas, incrementa los niveles de la glicemia a rangos menores que aquellos que permiten el diagnóstico de diabetes mellitus (DM). Estas elevaciones se han denominado glicemia de ayuno alterada (GAA) e intolerancia a la glucosa (ITG). Se asocian a una mayor incidencia de factores de riesgo cardiovascular tales como hipertensión arterial, dislipidemia y a una más frecuente aparición de DM. La transición de GAA o ITG a DM puede durar años, pero se estima que 70\% de ellos progresarán a dicho estado ${ }^{1}$.

El año 1995, un grupo de expertos patrocinado por la Sociedad de Diabetes de los Estados Unidos de Norteamérica (ADA) decidió cambiar los criterios diagnósticos de la DM enunciados en 1979²,3. Estos nuevos criterios se basaron en los niveles de glicemia de ayuno, definiendo los rangos de la glicemia de ayuno normal $<110 \mathrm{mg} /$ dl y crearon la categoría de GAA, la que fue definida como glicemia entre 110-125 mg/dl. Se estableció diagnóstico de DM cuando la glicemia en ayunas era $\geq 126 \mathrm{mg} / \mathrm{dl}$.

En ese criterio, se homologó GAA con ITG. Estudios posteriores demostraron que la medición de GAA subestimaba la incidencia de ITG $^{4}$. Ello llevó a recomendar el mantener el diagnóstico de ITG por carga de glucosa, ya que ésta se asocia a un riesgo cardiovascular superior al observado con GAA ${ }^{5}$.

En el año 2006 la ADA $^{6}$ estableció los siguientes criterios:

Normal. Glicemia de ayuno $<100 \mathrm{mg} / \mathrm{dl}$ y glicemia a $2 \mathrm{~h}$ de sobrecarga de glucosa $<140 \mathrm{mg} / \mathrm{dl}$.

GAA. Glicemia entre 100 y 125 mg/dl.

ITG. Glicemia en ayunas $<100 \mathrm{mg} / \mathrm{dl}$ y respuesta a las $2 \mathrm{~h}$ post-carga de glucosa entre 140 y $199 \mathrm{mg} / \mathrm{dl}$.

Glicemia en ayunas alterada e intolerancia a la glucosa. Glicemia en ayunas entre $100-125 \mathrm{mg} / \mathrm{dl}$ y a las 2 h de la carga de glucosa entre 140-199 mg/dl.

DM clínica. Glicemia en ayunas $\geq 126 \mathrm{mg} / \mathrm{dl}$ o a las $2 \mathrm{~h}$ de la carga de glucosa $\geq 200 \mathrm{mg} / \mathrm{dl}$.
La GAA y la ITG se asocian a un mayor riesgo de aparición de DM: para GAA sería entre 1-5\%/ año y 3-11\%/año para ITG, duplicándose las cifras cuando coexisten GAA+ITG ${ }^{7-9}$.

El significado clínico de GAA se ha modificado a través del tiempo, desde ser considerado como una expresión de la variabilidad técnica, a un estado evolutivo de la DM y por último como una situación independiente de la ITG, asociado a un mayor riesgo cardiovascular y de incidencia de DM clínica. Si bien ambas situaciones involucran resistencia insulínica, GAA se caracteriza fundamentalmente por una resistencia insulínica a nivel hepático, en cambio ITG lo es a nivel muscular. GAA tiene una fase aguda de secreción de insulina comprometida, pero una fase tardía normal, en cambio en ITG existe un severo déficit en la fase tardía. En algunos estudios se ha sugerido que GAA se asocia a un mayor compromiso de la función $\beta$ insular que ITG, lo que en el contexto de la historia natural de la DM 2, le conferiría un mayor riesgo de incidencia de DM clínica que ITG ${ }^{10-13}$. En el contexto de la prevención de DM, cuando se presentan estos estados precoces de disglicemias, se ha sugerido que las estrategias de prevención acorde ADA 2006 deben ser focalizadas en aquellos casos que presentan las condiciones conjuntas de GAA+ ITG. Ellos serían los candidatos más adecuados para la utilización de drogas insulino-sensibilizadores $^{6}$.

Dado todo lo anterior, hemos querido definir las características clínicas y metabólicas de los estados de GAA e ITG en una población chilena adulta de alto riesgo, con la finalidad de ayudar a entender mejor el significado de ambas condiciones a nivel de la práctica clínica.

\section{MATERIAL Y MÉTODO}

Población. Estudio retrospectivo realizado en 1.495 sujetos de ambos sexos, con edades entre 18 y 70 años. Se trata de una población de alto riesgo de DM que fue derivada por médicos para realizarse un test de sobrecarga a la glucosa en los laboratorios de la Pontificia Universidad Católica de Chile entre 2003 y 2005 (evaluación de los niveles de glicemia e insulinemia, en acuerdo a las normas de $\mathrm{ADA})^{6}$. 
Criterios de exclusión. Se excluyeron del análisis a los menores de 18 años $(n=42)$, a los mayores de 70 años ( $\mathrm{n}=27$ ), a los que tenían un IMC $\geq 50 \mathrm{Kg} / \mathrm{m}^{2}$ ( $\mathrm{n}=3$ ), y a los con insulinemia basal $\leq 2 \mu \mathrm{U} / \mathrm{ml}$ ( $\mathrm{n}$ 22). Todo lo anterior suma la exclusión de 91 sujetos, quedando incorporados al estudio 1.404 casos.

Métodos. Se efectuó el estudio de sobrecarga oral a la glucosa, previo ayuno de 8-12 h, con los pacientes en reposo. Se determinó glicemia e insulinemia de la sangre a tiempo 0 y a los 30, 60, 90 y 120 min después de una carga oral de 75 g de glucosa.

Se consignó, edad, sexo y los antecedentes de DM en familiares de primer grado. Se determinó el peso $(\mathrm{kg})$, la estatura corporal $(\mathrm{m})$ y se calculó el índice de masa corporal (IMC). Para esto último, se utilizó la formula clásica de Quatelêt: $\mathrm{IMC}=\mathrm{kg} / \mathrm{m}^{2}$.

La glicemia se determinó en sangre total según el método enzimático de la hexoquinasa en el equipo modular Hitachi, utilizando reactivos Roche. La insulina se determinó en plasma mediante el método de quimioluminiscencia (CLIA) en un equipo INMULITE 2000 DPC. Se estimó IR (índice de resistencia a la insulina), por HOMA-1, con la fórmula: IR $=$ glicemia $(\mathrm{mg} / \mathrm{dl}) \mathrm{x}$ insulinemia $(\mu \mathrm{U} / \mathrm{ml}) / 405$.

Se estimó la secreción insulínica (\% secreción $\beta)$, y el índice de disposición de la insulina (ID) o eficiencia de la función de células $\beta$, mediante el método computacional HOMA-2, diseñado en la Universidad de Oxford (Inglaterra), obtenido desde sitio de internet del proyecto (www.OCDEM.ox.ac.uk). El ID se obtuvo multiplicando porcentaje de secreción $\beta \mathrm{x}$ IR (expresado como porcentaje de sensibilidad) dividido por 100 y fue expresado en porcentaje.

En acuerdo a los criterios actuales de la $\mathrm{ADA}^{6}$, los sujetos fueron clasificados según los valores de glicemia en ayunas y a las $2 \mathrm{~h}$ post-carga de glucosa en:

- Normales

- Glicemia de ayunas alterada

- Intolerancia a la glucosa

- Glicemia de ayunas alterada e intolerancia a la glucosa

- Diabetes clínica

Se excluyeron del análisis del grupo GAA a aquellos casos con glicemia de ayunas entre $100 \mathrm{y}$ $125 \mathrm{~m} / \mathrm{dl}$ y con glicemias post carga de glucosa $\geq 140 \mathrm{mg} / \mathrm{dl}$. Se estimó como portador de resistencia insulínica a los sujetos que presentaban un HOMA-IR $\geq 2,53$, en acuerdo a las cifras comunicadas por Acosta et al ${ }^{14}$ para una población normal nacional. La función $\beta$ deteriorada fue categorizada a nivel de ID $\leq 60 \%$, por considerar a dicho nivel como el mejor punto discriminatorio entre normales y diabéticos clínicos en nuestra población.

Estadística. Debido a la gran dispersión de valores se procedió a normalizar el porcentaje secreción B, tomando como 100\% el valor de porcentaje secreción $\beta$ de la subpoblación de normales, calculando el resto de los valores en comparación a esta norma. Se utilizó estadística descriptiva para caracterizar las distintas subpoblaciones. Se utilizó $\mathrm{T}$ de Student, $\chi^{2}$ y ANOVA para comparar las variables de las distintas subpoblaciones.

\section{Resultados}

De 1.404 sujetos estudiados, $250(18,1 \%)$ eran hombres y $1.154(81,9 \%)$ mujeres, la edad promedio fue de 42,0 $\pm 14,2$ años.

El 63\% reportaron antecedentes de DM en familiares de primer grado, El IMC promedio fue de $28,1 \pm 5,0 \mathrm{~kg} / \mathrm{m}^{2}$. La glicemia basal 88,6 $\pm 11,3$ $\mathrm{mg} / \mathrm{dl}$, y a los 120 minutos post carga $106 \pm 38,3$. La insulinemia basal $10,6 \pm 6,7 \mu \mathrm{U} / \mathrm{ml}$ y a los 120 min post-carga $69,2 \pm 56,3 \mathrm{mg} / \mathrm{dl}$, el HOMA-IR fue $2,37 \pm 1,69$ y la eficiencia relativa de la función $\beta$ en el contexto de IR (ID), fue $102,4 \pm 35,8 \%$ (ver Tabla 1). De los 1.404 sujetos estudiados 1.097 $(78,1 \%)$ fueron categorizados como normales $(\mathrm{N})$, $106(7,5 \%)$ como con GAA, $103(7,3 \%)$ con ITG, $53(3,8 \%)$ con ITG + GAA, y por último $45(3,2 \%)$ como DM clínica (Tabla 2). Al analizar algunas características de los distintos estados de disglicemias, se observó que la población normal $(\mathrm{N})$ tenía significativamente menor edad ( $\mathrm{p}=<0,001)$, menor IMC ( $p=<0,001)$, y mayor ID $(p=<0,001)$ que las tres etapas identificadas como disglicemias.

El estado de glicemia alterada de ayuno (GAA) se caracterizó por presentar una sensibilidad insulínica (HOMA-IR) semejante a la población normal $2,0 \pm 1,3$ vs $2,0 \pm 2,2(p=0,204)$ y un ID significativamente menor $58 \pm 12 \%$ vs $111 \pm 32 \%$ 
Tabla 1. C aracterísticas generales de la población estudiada

\begin{tabular}{|ll|}
\hline Indicador & Promedio \\
\hline $\mathrm{n}$ & 1.404 \\
Edad (años) & $42,0 \pm 14,2$ \\
Sexo $(\mathrm{H} / \mathrm{M})$ & $250 / 1.154$ \\
Antecedentes familiares DM $(+) \%$ & 60,3 \\
$\mathrm{IMC}\left(\mathrm{Kg} / \mathrm{m}^{2}\right)$ & $28,1 \pm 5,0$ \\
Glicemia basal (mg/dl) & $88,6 \pm 11,3$ \\
Glicemia 120' post carga $(\mathrm{mg} / \mathrm{dl})$ & $106,4 \pm 38,3$ \\
Insulinemia basal $(\mu \mathrm{Ud} / \mathrm{ml})$ & $10,6 \pm 6,7$ \\
Insulinemia 120 ' post carga $(\mu \mathrm{Ud} / \mathrm{ml})$ & $69,3 \pm 56,3$ \\
HOMA-IR & $2,37 \pm 1,69$ \\
ID\% & $102,4 \pm 35,8$ \\
\hline
\end{tabular}

Tabla 2. N úmero y porcentaje de casos categorizados según diagnóstico

\begin{tabular}{|lrr|}
\hline Categoría & No casos & $\%$ \\
\hline Normal & 1.097 & 78,2 \\
GAA & 106 & 7,5 \\
GAA+ITG & 53 & 3,8 \\
ITG & 103 & 7,3 \\
DM clínica & 45 & 3,2 \\
Total & 1.404 & 100,0 \\
\hline
\end{tabular}

GAA + ITG = Glicemia de ayuno alterada + Intolerancia a la glucosa

( $\mathrm{p}=<0,001)$. En el estado de glicemia de ayuno alterada más intolerancia a la glucosa (GAA ITG), hubo un significativo mayor HOMA-IR 3,8 \pm 2,2 vs $2,0 \pm 2,2(\mathrm{p}=<0,001)$ y una discreta menor ID $57 \pm 10$ vs $58 \pm 11(\mathrm{p}=0,001)$ que en el de glicemia de ayuno alterada (GAA).

En la intolerancia a la glucosa (ITG) se observó significativamente mayor ID $96 \pm 26 \%$ que en GAA, GAA-ITG y DM ( $58 \pm 11 \%, 57 \pm 10 \%$ y $56 \pm 26 \%)$ respectivamente, aunque discretamente menor que en la población normal, $96 \pm$ $26 \%$ vs $111 \pm 32 \%$ ( $p=0,001)$. Además se apreció una mayor resistencia insulínica que en la población normal y la portadora de glicemia de ayuno alterada $(\mathrm{p}<0,001)$.

En los diabéticos clínicos (DM) se destacó una significativa mayor edad, IMC, HOMA-IR y un menor ID ( $56 \pm 26 \%)$ en comparación con GAA, GAA - ITG e ITG. No hubo diferencia en la tasa de antecedentes familiares con DM entre los diferentes grupos estudiados

En la Figura 1 se presenta el porcentaje de casos identificados en las distintas categorías clínicas que presentaban resistencia insulínica (HOMA-IR $\geq 2,53$ ) o una función $\AA$ inadecuada (ID $\leq 60 \%$ ).

Se aprecia que si bien en la población normal existía $36,7 \%$ de sujetos con resistencia insulínica, sólo 3,2\% presentaban una función $B$ inadecuada y en cambio en los tres estados de disglicemia existió una significativa diferencia en la proporción de casos con resistencia a la insulina; 57,5 a $75 \%$, y en el porcentaje con función $ß$ inadecuada $30,1 \%$ en ITG, $62,0 \%$ en GAA y $89,0 \%$ en DM. 
Tabla 3. Algunas características de las distintas categorías de intolerancia a la glucosa

\begin{tabular}{|c|c|c|c|c|c|}
\hline & Normal & GAA & GAAIG & ITG & DM \\
\hline $\mathrm{N}$ & 1.097 & 106 & 53 & $103^{\neq}$ & 40 \\
\hline Edad ( años) & $39 \pm 13^{*}$ & $51 \pm 12^{*}$ & $52 \pm 12^{*}$ & $49 \pm 12^{*}$ & $57 \pm 13^{*}$ \\
\hline Antecedentes familares (+) & $61 \%$ & $57 \%$ & $55 \%$ & $60 \%$ & $65 \%$ \\
\hline$\%$ de mujeres & $87 \%$ *** & $57 \%$ *** & $66 \% * *$ & $79 \% * *$ & $64 \% * *$ \\
\hline $\operatorname{IMC}\left(\mathrm{Kg} / \mathrm{m}^{2}\right)$ & $28 \pm 4,9 \vee$ & $30 \pm 5,0 \diamond$ & $30 \pm 4,7 \diamond$ & $29 \pm 4,4 \diamond$ & $31 \pm 5,7 \diamond$ \\
\hline HOMA-IR & $2.0 \pm 1,3^{\dagger}$ & $2,0 \pm 0,60^{\dagger}$ & $3,8 \pm 2,20^{\dagger}$ & $2,6 \pm 1,5^{\dagger}$ & $4,4 \pm 3,7^{\dagger}$ \\
\hline ID\% & $111 \pm 32 \%^{* * * *}$ & $58 \pm 11 \% \%^{* * *}$ & $57 \pm 10 \% * * *$ & $96 \pm 26 \% * * *$ & $56 \pm 26 \% \%^{* * * *}$ \\
\hline
\end{tabular}

*Normal diferente de todos los otros grupos ( $\mathrm{p}<0.000 \mathrm{vs}$ el resto). GAA no es diferente de GAAIG. (+) Sin diferencias entre grupos en tasa de antecedentes familiares. **Porcentaje de mujeres normales mayor que todos los otros grupos. En los estados alterados del metabolismo, el mayor porcentaje de mujeres se observa en ITG. VIMC de normales menor que todos los otros grupos $(p<0,0000)$. Sin diferencias entre los otros grupos. ${ }^{\dagger}$ Homa normales menor que el resto de los grupos ( $p<0,000$ ), IG $>$ GAA y IG $<$ GAAIG $/ \mathrm{p}<0.00)^{* * *}$ ID mayor en normales que el resto de los grupos ( $\left.\mathrm{p}<0,004\right)$. IG mayor en GAA que en GAAIG $(\mathrm{p}<0,000)$. DM difiere de GAA y GAAIG sólo en edad ( $\mathrm{p} \leq 0,04$ y 0,004 , respectivamente. DM difiere de $(\mathrm{p}<0,000)$, IMC $(\mathrm{p}<0,015)$, HOMAIR $(\mathrm{p}<0,000)$ e ID $(\mathrm{p}<0,000)$.

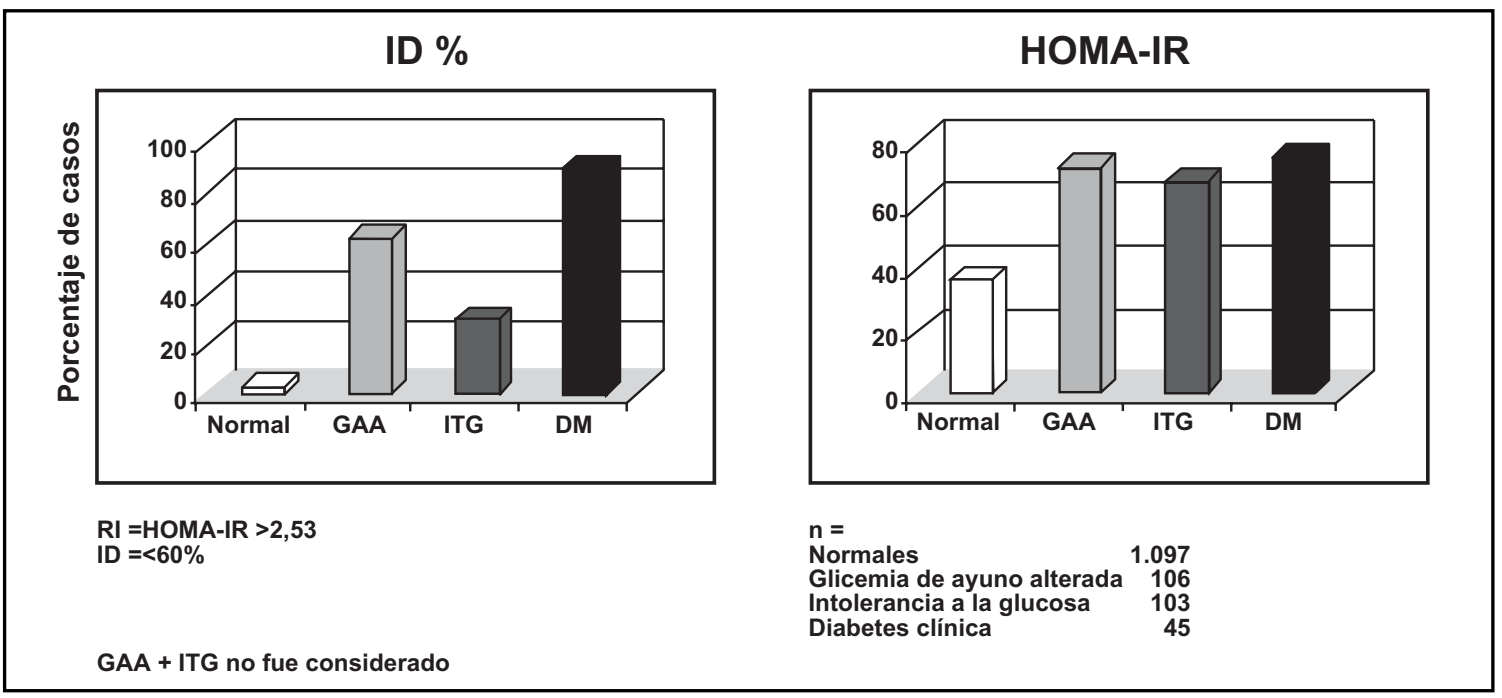

Figura 1. Porcentaje de individuos con resistencia insulínica (RI) y función crítica de células ß (ID).

DISCUSIÓN

La prevalencia comunicada de GAA y de ITG varía ampliamente a nivel internacional, entre 4\%-26\% de GAA y entre 10\%-15\% de ITG y de $1-3 \%$ de coexistencia de GAA-ITG ${ }^{15-17}$. Como factores condicionantes de estas variaciones en la prevalencia, se han identificado: al origen étnico, al sexo (ITG más prevalente en mujeres), la edad (prevalencia creciente con la edad, especialmente GAA + ITG), y problemas de diseño debido a la utilización de diferentes criterios diagnósticos. Igual hecho sucede con la predicción de riesgo. La mayoría de los estudios señalan que ambas condiciones confieren 
un discreta tendencia aunque significativa e independiente de asociarse a factores de riesgo cardiovascular, lo que es más significativo para ITG $^{8,9}$.

Nuestro estudio de diseño transversal no nos permite discutir acerca de la evolutividad de las categorías de disglicemias. Por otro lado, por tratarse de una población de alto riesgo con $~ 60 \%$ de antecedentes familiares directos de DM2, no representa a la población general, pero sí permite identificar la prevalencia de los distintos estados de disglicemia identificados y describir sus características clínicas.

Un hecho destacado es la confirmación del estado de glicemia de ayuno alterada (GAA), con una prevalencia aislada de 7,5\% (106 casos) y asociada a Intolerancia a la glucosa (GAA-ITG) en 3,8\% (53 casos).

GAA difiere de ITG en varios sentidos. Sólo coexiste con ITG en 53 casos de un total de 156 (34\% del total de GAA), se presenta a edad mayor, el nivel de resistencia a la insulina es discretamente menor al observado en los sujetos catalogados como normales, pero su función $ß$ se asemeja al de los diabéticos clínicos.

La asociación de GAA + IG se asemeja a las características fisiopatológicas de la DM clínica: mayor resistencia insulínica y deterioro de la función $ß$ que en GAA. Esto justifica el consenso de la ADA y AAEC, que define a esta condición como la de mayor riesgo de DM clínica, y sugiere focalizar en estos casos el uso de insulinosensibilizadores. Como ya se señaló, el diseño transversal no hace posible definir una secuencia patológica. Nuestros datos presentan a GAA como un estado que precede a las manifestaciones clínicas de la DM, ya que en ella se observa una significativa pérdida de la capacidad de las células $\beta$ para compensar una resistencia insulínica determinada. Sin embargo no es posible descartar que

\section{REFERENCIAS}

1. KAHN SE. The relative contribution of insulin resistance and $\beta$ cell disfunction to the pathophysiology of type 2 Diabetes. Diabetologia 2003; 46: 3-9.

2. Nathan DM, Davidson M, De Fronzo R, Heine RR, Pratley $\mathrm{R}$, ZINMAN B. Impaired fasting glucose and impaired glucose tolerance Diabetes Care 2007; 30: 753-8.

3. National Diabetes Data Group. Classification and diagnosis of Diabetes Mellitus and other catego- ambas condiciones, GAA e ITG, representen estadios de etiología diferente.

En el momento actual existen evidencias que es posible retrasar la aparición de la DM tipo 2 clínica en la etapa de ITG, mediante cambios de estilo de vida o utilizando fármacos insulinosensibilizadores $^{18}$. No existen estudios de este tipo en relación a GAA. Por el momento parece prudente evaluar clínicamente a los portadores de GAA, y enfrentar y corregir aquellos factores que puedan estar involucrados en su génesis. Más aún, recientemente el consenso AACE/ADA $2008^{18}$ ha recomendado que los objetivos del manejo de la "prediabetes" para lograr reducir la incidencia cardiovascular en el DM, deben ser los mismos que los de la DM clínica: hemoglobina glicosilada A1c $<7 \%$, C-LDL $\leq 100 \mathrm{mg} / \mathrm{dl}$ y presión arterial $\leq 130 / 80 \mathrm{mmHg}$. La interpretación de un nivel de glicemia de ayunas elevado puede ser difícil en la clínica, ya que las variaciones pueden deberse a la variabilidad técnica. Como una solución se ha sugerido la repetición de la medición y sólo cuando la disglicemia se confirma, se estaría autorizado para catalogar al enfermo.

Sin embargo, en muchas ocasiones el sujeto, conciente o inconcientemente, realiza limitaciones que pueden interferir con los exámenes subsecuentes. Parece recomendable advertir al paciente de esta eventualidad y solicitar una segunda medición en todos los casos de GAA, además de una prueba de sobrecarga a la glucosa, para poder identificar los casos de mayor riesgo, aquellos con ITG asociada, y decidir el uso de insulinosensibilizadores, de preferencia metformina.

A partir de nuestras observaciones, en concordancia con los consensos actuales, el clínico debe identificar a la glicemia de ayuno alterada como una manifestación del síndrome diabético y enfrentarla como tal.

ries of glucose intolerance. Diabetes 1979; 28 : $1039-57$.

4. Expert Committee on the diagnosis and Classification of Diabetes Mellitus. 1997 "Report of the expert committee on the diagnosis and classification of Diabetes Mellitus". Diabetes Care 1997; 20: 1138-97.

5. Genuth S, Alberti KG, Bennet P, Buse J, De Fronzo R, KAHN R ET AL. Follow-up report on the diagnosis of Diabetes Mellitus. Diabetes Care 2003; 26: 3160-7.

6. American Diabetes Association. Standards of medical 
care in Diabetes 2006. Diabetes Care 2006; 29 (Suppl 1): $S 4-S 42$.

7. Rasmussen SS, Glumer C, Lauritzen T, Borch-Jonson K. Progression from impaired fasting glucose and impaired glucose tolerance to Diabetes in a High Risk screening programme in general practice: the ADDITION Study Denmark. Diabetologia 2007; 50: 293-7.

8. Meigs JB, Nathan DM, D'Agostino RB, William PW. Fasting and Post-challenge glicemia and cardiovascular disease risk The Framingham Offpring Study. Diabetes Care 2002; 25: 1845-50.

9. Tominaga M, Eguchi H, Manaka H, Igarashi K, Kato T, SeKIKAwa A. Impaired glucose tolerance is a risk factor for cardiovascular disease, but not impaired fasting glucose. The Funagata Diabetes Study. Diabetes Care 1999; 22: 920-24.

10. Weyer C, Bogardus C, Pratley RE. Metabolic characteristics of individual with impaired fasting glucose and/or impaired glucose tolerance. Diabetes 1999; 48; 2197-203.

11. Davies MJ, Raymond NT, Day Ji, Holes CN, Burdem AC ET AL. Impaired glucose tolerance and impaired fasting hyperglycaemia have different characteristics. Diab Med 2000; 17: 433-40.

12. Tripathy D, Carlsson M, Almgren P, Isomaa B, Taskinen MR, Tuomi T, Groop LC. Insulin secretion and insulin sensibility in relation to glucose intolerance. Lessons from the Botnia Study. Diabetes 2000; 49: 975-80.
13. Festa A, D'agostino R, Hanley A, Karter A, SaAd M, HafFner $S$ ET aL. Differences in insulin resistance in non diabetic subjects with impaired glucose tolerance or isolated impaired fasting glucose. Diabetes 2004; 53: 1549-55.

14. Acosta Am, escalona M, Maiz A, Pollak F, Leighton F. Determinación del índice de resistencia insulínica mediante HOMA en una población de la región Metropolitana de Chile. Rev Méd Chile 2002; 130: 1227-31.

15. Quiao Q, Nakagami T, Tuomilehto J, Borch-Johnsen K, BalkaW B, Iwamoto et al. International Diabetes Epidemiological Group. DECODA Study Group: Comparition of the fasting and 2 hour glucose criteria for Diabetes states in Different Asian Cohorts. Diabetologia 2000; 43: 1470-7.

16. Gu D, Reynolds K, Duan X, Xin X, Chen J, Wu X et al. Prevalence of diabetes and impaired fasting glucose in the Chinese adult population. International Collaborative Study of Cardiovascular disease in Asia (InterASIA) Diabetologia 2003; 46: 1190-8.

17. Cowie CC, Rust KF, Byrd-Holt DD, Eberhardt MS, Flegal KM, Engelgau MM ET al. Prevalence of Diabetes, impaired fasting glucose and impaired glucose tolerance in US adults: The National Health and Nutrition Examination Survey 1999-2002. Diabetes Care 2006; 29: 1263-8.

18. AaCE Diabetes Mellitus Guidelines. Endocr Practic 2007; 13 (Suppl 1) : 1-64.

\section{Glosario}

$\begin{array}{ll}\text { DM } & \text { : Diabetes Mellitus } \\ \text { GAA } & \text { : Glicemia de ayuno alterada } \\ \text { ITG } & \text { : Intolerancia a la glucosa } \\ \text { IMC } & \text { : Índice de masa corporal } \\ \text { IR } & : \text { Resistencia insulínica } \\ \text { HOMA } & \text { : Homeostatic Model Assesment } \\ \text { HOMA-IR } & \text { : Indicador de sensibilidad insulínica } \\ \text { ADA } & \text { : American Diabetes Association } \\ \text { AACE } & \text { : American Association Clinical Endocrinologist }\end{array}$

